\title{
Ventilation inhomogeneity in patients with cystic fibrosis measured by electrical impedance tomography
}

\author{
Zhanqi Zhao ${ }^{1}$, Rainald Fischer ${ }^{2}$, Ullrich Müller-Lisse ${ }^{3}$, Knut Moeller ${ }^{1}$ \\ ${ }^{1}$ Department of Biomedical Engineering, Furtwangen University, Villingen-Schwenningen, Germany; zhanqi.zhao@hs- \\ furtwangen.de \\ ${ }^{2}$ Department of Pneumology, University of Munich, Munich, Germany \\ ${ }^{3}$ Department of Radiology, University of Munich, Munich, Germany
}

\begin{abstract}
Objective: The distribution of ventilation within the lung is inhomogeneous. We hypothesized that the degree of inhomogeneity in patients with cystic fibrosis (CF) differs from that in healthy subjects.

Method: Three adult patients with cystic fibrosis (CF) and two healthy subjects were recruited for this preliminary study. Subjects were asked to breathe in as deep and exhale as hard as possible three times. Electrical impedance tomography (EIT) measurements were performed during spirometry tests. The global inhomogeneity index(GI) was applied to assess the degree of ventilation homogeneity at $25 \%, 50 \%, 75 \%$ and $100 \%$ of maximum inspiratory volume.

Results: Airway obstruction was detected in CF patients but not in healthy volunteers during spirometry tests. GI decreased as inspiratory volume increased in CF patients $(0.59 \pm 0.20$ at $25 \%$ and $0.39 \pm 0.09$ at $100 \%, \mathrm{p}<0.01, \mathrm{mean} \pm \mathrm{SD})$ while GI remained unchanged in healthy subjects $(0.40 \pm 0.05$ at $25 \%$ and $0.37 \pm 0.03$ at $100 \%, p=0.31)$.

Conclusion: Degree of ventilation homogeneity appears to vary during forced respiration in CF patients, but not in healthy volunteers. EIT is a practical method to measure the inhomogeneity of ventilation distribution.
\end{abstract}

\section{Introduction}

Cystic fibrosis (CF) is an inherited, chronic disease of the body's mucus and sweat glands that mainly affects the lungs and the digestive system. The alteration of lung structure and small airways leads to flow reduction during in- and expiration [1-2]. There is still no cure for CF, and it tends to increase in severity over time [3]. Despite improved treatment, mean survival time of $\mathrm{CF}$ patients is only 37 years [4].

Distribution of tidal volume in lungs is often inhomogeneous. The degree of inhomogeneity reveals information about the patients' respective lung conditions. The routine diagnosis tools for CF patients such as spirometry and body plethysmography are unable to assess ventilation distribution. Other methods such as multibreath washout technique and computed tomography (CT) are inconvenient or even potentially harmful to the patient and therefore not suitable for outpatients.

Electrical Impedance Tomography (EIT) is a noninvasive, radiation-free imaging technique, which can monitor both regional lung ventilation and tidal volume distribution, by measuring the electrical potentials at the chest wall surface. The rationale is that changes in regional air content and regional blood flow modify the electrical impedance of lung tissue [5-6]. We have recently developed an EITbased global inhomogeneity (GI) index to assess ventilation inhomogeneity [7]. We hypothesized that due to disease-related airway obstruction, ventilation inhomogeneity in $\mathrm{CF}$ patients varies during forced respiration and it can be detected with EIT.

\section{Patients and Methods}

Three patients with cystic fibrosis (CF) (1 male and 2 female; body height, 180, 165 and $156 \mathrm{~cm}$; body weight, 64, 58 and $55 \mathrm{~kg}$; age at examination, 32, 35 and 30 years, respectively) and two healthy volunteers (2 male; body height, 178 and $168 \mathrm{~cm}$; body weight, 62 and $60 \mathrm{~kg}$; age at examination, 50 and 28 years, respectively) were investigated. Written informed consent was obtained from all patients prior to the study. The study was approved by the local ethics committee.

All subjects were asked to breathe in as deep and exhale as hard as possible three times at the spirometer, according to standard ATS spirometry guidelines [8]. An EIT electrode belt, which carries 16 electrodes with a width of $40 \mathrm{~mm}$, was placed around the chest in the fifth intercostal space while one reference electrode was placed on the patients' abdomen (EIT Evaluation KIT 2, Dräger Medical, Lübeck, Germany). EIT images were continuously recorded at 20 $\mathrm{Hz}$ and stored during spirometry tests.

GI index has been described in previous studies [7, 9]. In this preliminary study, the GI index was slightly modified to evaluate the inhomogeneity degree at $25 \%, 50 \%, 75 \%$ and $100 \%$ of maximum inspiratory volume. For every forced respiration, four difference images, which represent the respective differences of impedance between $25 \%$, $50 \%, 75 \%, 100 \%$ of maximum inspiratory volume and inspiration begin $(0 \%)$, were generated. Lung regions in EIT images were defined using the LAE method [10]. The median value of the pixels in the lung area is calculated for each difference image. The GI index is defined as the sum 
of the absolute differences between the median value and every pixel value, which is normalized with the sum of the impedance values within the lung area:

$$
G I=\sum_{x, y \in \text { lung }}\left|D I_{x y}-\operatorname{Median}\left(D I_{\text {lung }}\right)\right| / \sum_{x, y \in \text { lung }} D I_{x y}
$$

where $D I$ is the value of the differential impedance in the difference images; $D I_{x y}$ denotes the pixel in the identified lung area; $D I_{\text {lung }}$ are all pixels in the lung area under observation.

Data analysis was performed using MATLAB (The MathWorks Inc., Natick, MA, USA). Lilliefors test was used to confirm the distribution of the data. In case of normal distribution, results were expressed as mean \pm SD. Unpaired t-test was used to evaluate the difference of GI index between level $25 \%$ and $100 \%, 50 \%$ and $100 \%, 75 \%$ and $100 \%$. $\mathrm{P}<0.05$ was considered statistically significant. The significant levels were corrected for multiple comparisons using Holm's sequential Bonferroni method.

\section{$3 \quad$ Results}

Figure 1 shows the ventilation distribution during one forced inspiration-expiration cycle and one normal spontaneous breath in a CF patient. According to the GI index, air distribution was more homogeneous during forced breathing than during spontaneous breathing.

When the forced breathing cycle was analyzed at 4 different levels, the GI index decreased as inspiratory volume increased in CF patients, while it remained unchanged in healthy subjects (Table 1).

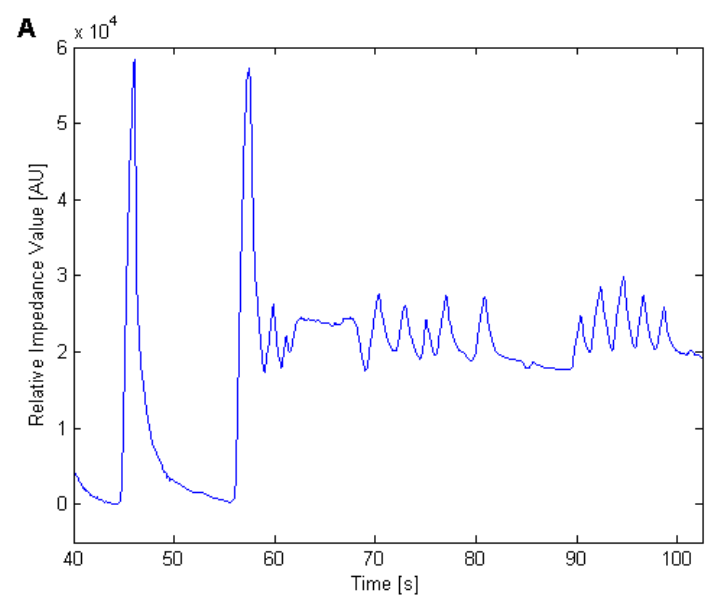

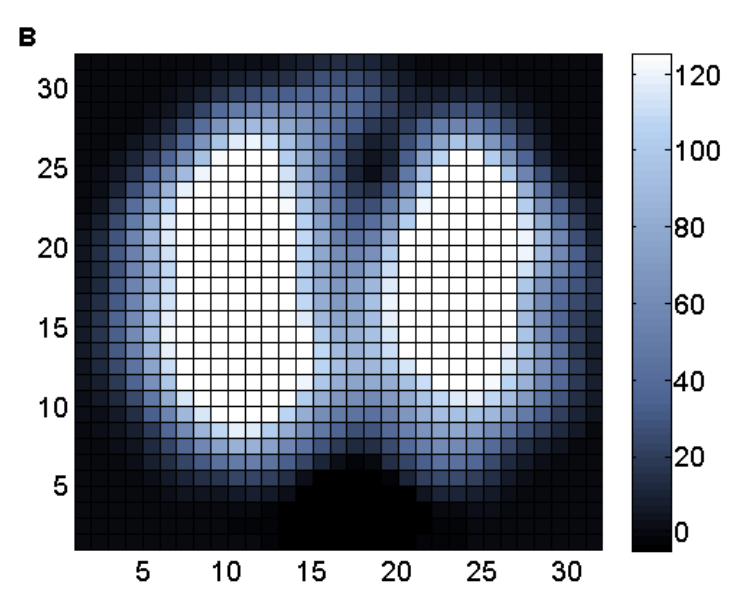

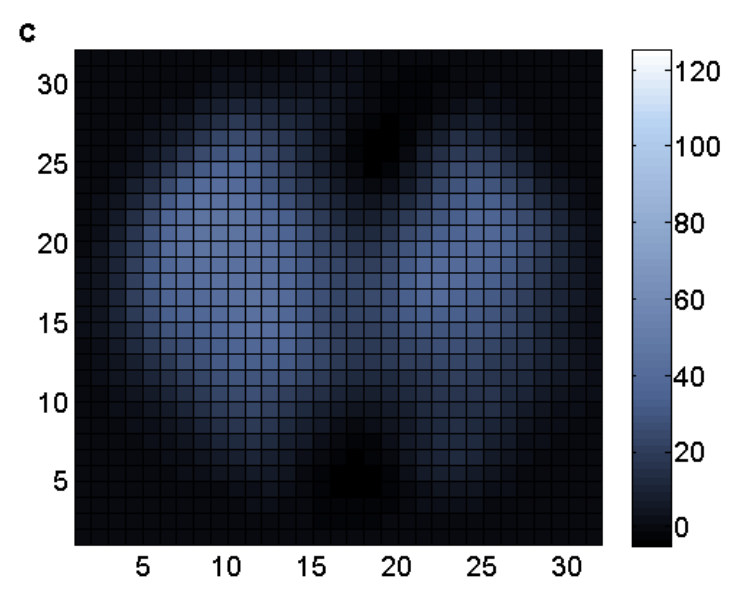

Fig. 1 Typical global impedance curve (A) and tidal images of EIT during forced breathing (B) and normal, spontaneous breathing $(\mathrm{C})$ in one $\mathrm{CF}$ patient. AU: arbitrary unit.

Table 1 GI index of CF patients and healthy volunteers at different levels of maximum inspiratory volume.

\begin{tabular}{|l|l|l|}
\hline Subjects Category & CF & Healthy \\
\hline $25 \%$ & $0.59 \pm 0.20 *$ & $0.40 \pm 0.05$ \\
\hline $50 \%$ & $0.49 \pm 0.15$ & $0.36 \pm 0.03$ \\
\hline $75 \%$ & $0.43 \pm 0.12$ & $0.35 \pm 0.02$ \\
\hline $100 \%$ & $0.39 \pm 0.09 *$ & $0.37 \pm 0.03$ \\
\hline$*$ p-value & $\mathrm{P}<0.01$ & \\
\hline
\end{tabular}

Figure 2 shows the difference images of one CF patient at different preselected levels of the forced breathing cycle. In this particular patient, the right lung (on the left side of EIT images) was inhomogeneously ventilated at $25 \%$ and $50 \%$ of the maximum inspiratory volume. Ventilation distribution became more homogeneous at higher inspiratory volume levels. 
A

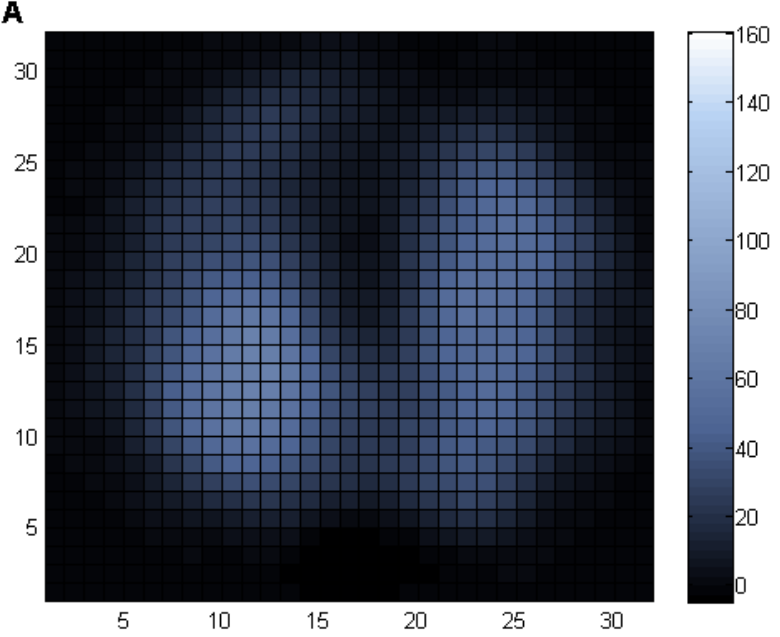

B

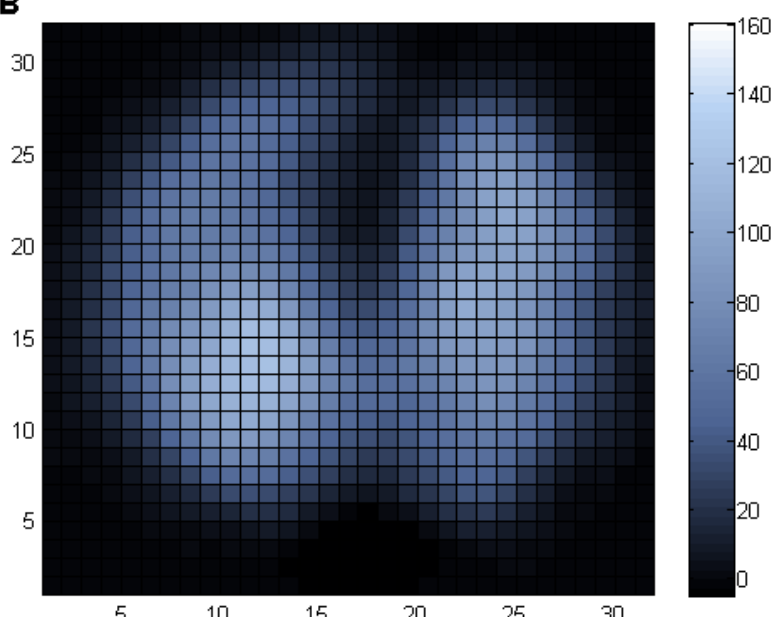

C

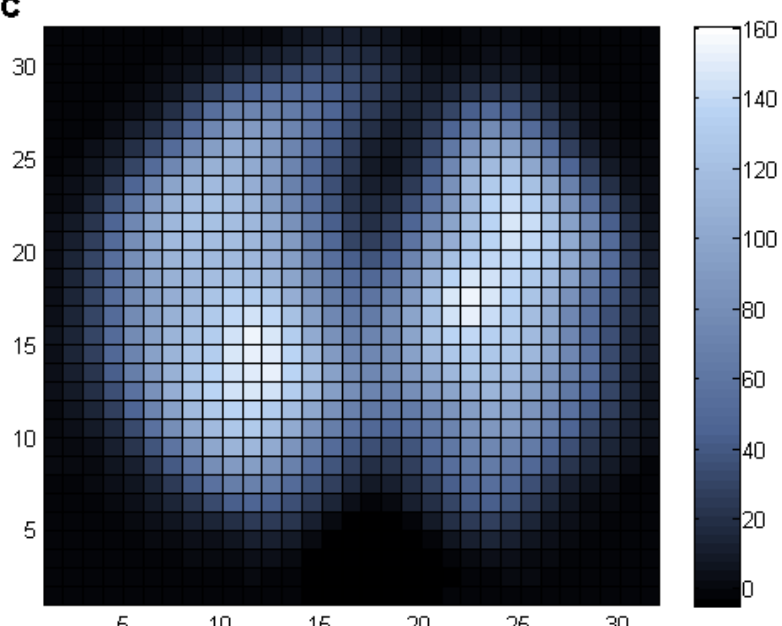

D

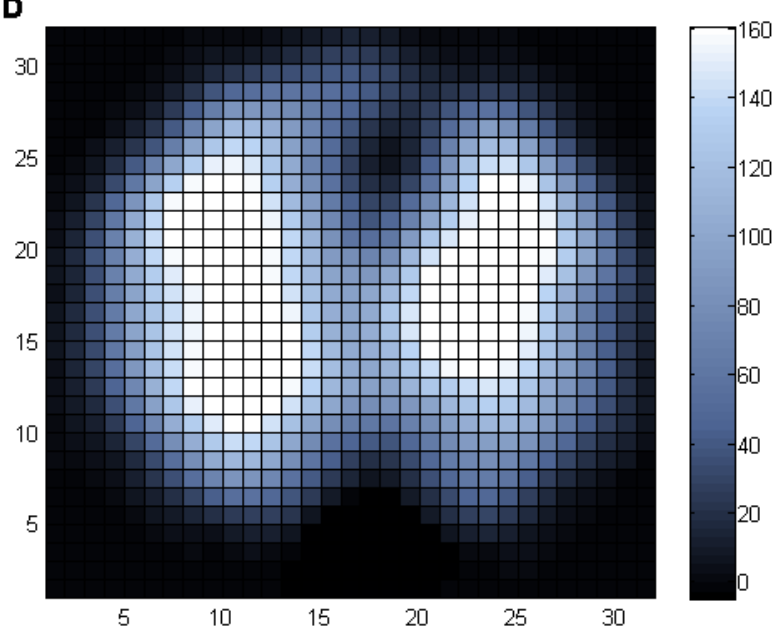

Fig. 2 Difference images of one CF patient at $25 \%$ (A), $50 \%$ (B), $75 \%$ (C) and $100 \%$ (D) of maximum inspiratory volume.

\section{Discussion}

In this preliminary study of 5 spontaneously breathing subjects, EIT, as applied during lung function tests demonstrated that ventilation homogeneity differed between CF patients and healthy subjects during forced respiration. Since its early application in the 1980s [11], EIT analysis of the inhomogeneity of air distribution within the lungs has attracted the attention of researches all over the world [7]. However, methods were missing which could both simplify the complex information provided by EIT, and consider the contribution of every pixel in EIT images [7]. Hence, we defined the GI index to quantify tidal volume distribution within the lung. The reliability and potential use of the GI index for mechanically ventilated patients has previously been investigated [7,9]. The GI index has also been applied for spontaneously breathing subjects in different body positions [12].

Differences in ventilation homogeneity between healthy volunteers and patients with critical lung disease have previously been reported [7]. In theory, the degree of ventilation inhomogeneity should be relatively low for CF patients when compared to patients with critical lung disease such as acute respiratory distress syndrome. Therefore, it should be more difficult to distinguish patients with $\mathrm{CF}$ from healthy volunteers by means of EIT. However, due to chronic airway obstruction in CF patients, it may take longer for air to distribute within their lungs. During cycles of forced inspiration and expiration, there should be more time for air distribution. Thus we assessed the ventilation distribution with EIT during lung function tests, with recording of three subsequent cycles of forced breathing. As we hypothesized, the EIT-based GI index demonstrated ventilation inhomogeneity at the onset of forced breathing in $\mathrm{CF}$ patients, while ventilation became more homogeneous during later phases of inspiration. No significant changes of ventilation over the breathing cycle were found in healthy volunteers. 
A limitation of the present study is that only three patients with CF and two healthy subjects were included. To corroborate these early results, more subjects will be examined by means of EIT.

\section{Conclusion}

The degree of ventilation homogeneity varies during forced respiration in CF patients. The EIT-based GI index appears to be a practical method to measure the inhomogeneity of ventilation distribution.

\section{Acknowledgment}

This work was partially supported by Bundesministerium für Bildung und Forschung (Grant 1781X08 MOTiF-A), and Dräger Medical, Lübeck.

\section{References}

[1] Bitterling H: Imaging of patients with Mucoviscidosis. In: Multi slice CT. Edited by Reiser M, Takahashi M, Modic M, Becker C, 2. edn. Berlin: Springer Verlag; 2004: 131-136.

[2] Maffessanti M, Candusso M, Brizzi F, Piovesana F: Cystic fibrosis in children: HRCT findings and distribution of disease. J Thorac Imaging 1996, 11(1):27-38.

[3] Fischer R, Nahrig S, Kappler M, Griese M: [Cystic fibrosis care in transition from adolescence to adult age]. Internist (Berl) 2009, 50(10):1213-1214, 1216, 1218-1220.

[4] Torpy JM, Lynm C, Glass RM: JAMA patient page. Cystic fibrosis. JAMA 2009, 302(10):1130.

[5] Nopp P, Rapp E, Pfutzner H, Nakesch H, Ruhsam C: Dielectric properties of lung tissue as a function of air content. Physics in medicine and biology 1993, 38(6):699-716.

[6] Brown BH, Barber DC, Morice AH, Leathard AD: Cardiac and respiratory related electrical impedance changes in the human thorax. IEEE transactions on bio-medical engineering 1994, 41(8):729-734.

[7] Zhao Z, Moller K, Steinmann D, Frerichs I, Guttmann $\mathrm{J}$ : Evaluation of an electrical impedance tomography-based global inhomogeneity index for pulmonary ventilation distribution. Intensive care medicine 2009, 35(11):1900-1906.

[8] Miller MR, Hankinson J, Brusasco V, Burgos F, Casaburi R, Coates A, Crapo R, Enright P, van der Grinten CP, Gustafsson P et al: Standardisation of spirometry. Eur Respir J 2005, 26(2):319-338.

[9] Zhao Z, Steinmann D, Frerichs I, Guttmann J, Moller $\mathrm{K}$ : PEEP titration guided by ventilation homogeneity: a feasibility study using electrical impedance tomography. Critical care (London, England) 2010, 14(1):R8

[10] Zhao Z, Steinmann D, Muller-Zivkovic D, Martin J, Frerichs I, Guttmann J, Moller K: A lung area estimation method for analysis of ventilation inhomogeneity based on electrical impedance tomography. J Xray Sci Technol 2010, 18(2):171-182.

[11] Barber DC, Brown BH: Applied potential tomography. J Phys E: Sci Instrum 1984, 17:723-733.

[12] Zhao Z, Kiefer N, Kulcsar M, Lang S, Möller K: Ventilation inhomogeneity in upright and supine position assessed by electrical impedance tomography. In: MEDICON 2010, IFMBE Proceedings: 2010; Chalkidiki, Greece: Springer; 2010. 